\title{
Chromosome instability is common in human cleavage-stage embryos
}

\author{
Evelyne Vanneste ${ }^{1,2,9}$, Thierry Voet ${ }^{1,9}$, Cédric Le Caignec ${ }^{1,3,4}$, Michèle Ampe ${ }^{5}$, Peter Konings ${ }^{6}$, Cindy Melotte ${ }^{1}$, \\ Sophie Debrock ${ }^{2}$, Mustapha Amyere ${ }^{7}$, Miikka Vikkula ${ }^{7}$, Frans Schuit ${ }^{8}$, Jean-Pierre Fryns ${ }^{1}$, Geert Verbeke ${ }^{5}$, \\ Thomas D'Hooghe ${ }^{2}$, Yves Moreau ${ }^{6}$ \& Joris R Vermeesch ${ }^{1}$
}

\begin{abstract}
Chromosome instability is a hallmark of tumorigenesis. This study establishes that chromosome instability is also common during early human embryogenesis. A new array-based method allowed screening of genome-wide copy number and loss of heterozygosity in single cells. This revealed not only mosaicism for whole-chromosome aneuploidies and uniparental disomies in most cleavage-stage embryos but also frequent segmental deletions, duplications and amplifications that were reciprocal in sister blastomeres, implying the occurrence of breakagefusion-bridge cycles. This explains the low human fecundity and identifies post-zygotic chromosome instability as a leading cause of constitutional chromosomal disorders.
\end{abstract}

Chromosome instability is characterized by an elevated rate of gains or losses of complete chromosomes or segments of chromosomes per cell cycle resulting in cell-to-cell variability ${ }^{1}$. Aside from the frequent occurrence of chromosome instability in human malignant solid tumors $^{2-4}$, chromosome instability has not been implicated in other biological processes. Nevertheless, the numerous studies reporting mosaic whole-chromosome imbalances ${ }^{5}$ and the sporadic reports on rare segmental aneuploidies ${ }^{6-8}$ in human in vitro fertilized (IVF) embryos insinuate that chromosome instability might occur during human embryogenesis as well. As most of these studies rely on interphase fluorescent in situ hybridization (FISH) targeting only a few loci or on low-resolution comparative genomic hybridization, a comprehensive view of the genomic imbalances is lacking. Moreover, because the majority of the studies are performed on embryos obtained from couples considered at risk for embryonic aneuploidy (with, for example, advanced maternal age, recurrent implantation failure, recurrent miscarriages or severe male factor infertility, which are the main indications for preimplantation genetic aneuploidy screening $)^{9}$, little is known about chromosomal imbalances in embryos from normal fertile women.

To investigate embryonic chromosome instability and to unravel its frequency, nature and consequences, we developed two new arraybased approaches to explore the genomes of single cells ${ }^{10-12}$; in combination, the approaches increase the accuracy of copy number variation $(\mathrm{CNV})$ detection. We applied this technique to analyze the genomic constitution of all available blastomeres from 23 good-quality embryos from young women ( $<35$ years old) without preimplantation genetic aneuploidy screening indications undergoing IVF for genetic risks not related to fertility (Supplementary Table 1 online). This analysis unexpectedly revealed a high frequency of chromosome instability in cleavage-stage embryos involving complex patterns of segmental chromosomal imbalances and mosaicism for whole chromosomes and uniparental isodisomies. These patterns are reminiscent of the chromosomal instabilities observed in human cancers ${ }^{1}$ and correspond with the complex chromosomal aberrations observed in individuals with birth defects ${ }^{13-15}$.

\section{RESULTS}

Single-cell CNV and single nucleotide polymorphism detection

First, we co-hybridized amplified DNA from a single cell and differentially labeled, nonamplified genomic DNA (with karyotype $47, \mathrm{XXY}$ ) on a bacterial artificial chromosome (BAC) microarray $^{10,11,16}$. A new algorithm corrected intensity ratios for clone-specific amplification biases and subsequently determined for each clone a smoothed posterior probability of being normal diploid, deleted and duplicated in the analyzed single cell. This algorithm also provided a quality control for single-cell array data, because equal probabilities for the three copy number states across the genome indicated that an imbalance could not be statistically discriminated from the other copy number states. Second, we hybridized single-cell amplified DNA to the single nucleotide polymorphism (SNP) array. Copy number analysis of the SNP-probe intensities by the five-state hidden Markov model allowed not only independent confirmation of the genomic imbalances detected with BAC array comparative genomic hybridization (CGH), but also detection of nullisomic and amplified DNA segments. In addition, single-cell genotypes were determined. Both uniparental isodisomy and hemizygous loci are characterized by the absence of heterozygosity, henceforth termed loss of heterozygosity $(\mathrm{LOH})$.

${ }^{1}$ Center for Human Genetics, K.U.Leuven, Leuven, Belgium. ${ }^{2}$ Leuven University Fertility Center, University Hospital Gasthuisberg, Leuven, Belgium. ${ }^{3}$ Centre Hospitalier Universitaire de Nantes, Service de Génétique Medicale, Nantes Cedex, France. ${ }^{4}$ Institut National de la Santé et de la Recherche Médicale, I'Institut du Thorax, Nantes, France. ${ }^{5}$ Interuniversity Institute for Biostatistics and Statistical Bioinformatics, K.U.Leuven, Leuven, Belgium. ${ }^{6}$ ESAT-SISTA, K.U.Leuven, Heverlee, Belgium. ${ }^{7}$ de Duve Institute, Université Catholique de Louvain, Brussels, Belgium. ${ }^{8}$ Molecular Cell Biology, Gene Expression Group, University Hospital Gasthuisberg, Leuven, Belgium. ${ }^{9}$ These authors contributed equally to this work. Correspondence should be addressed to J.R.V. (joris.vermeesch@uz.kuleuven.be). 


\begin{tabular}{|c|c|c|c|c|c|c|c|}
\hline \multirow[b]{2}{*}{ Karyotype of the lymphoblastoid cells } & \multirow[b]{2}{*}{ Imbalances } & \multicolumn{3}{|c|}{$\begin{array}{c}\text { Number of probes within the imbalance detected } \\
\text { with reference genomic DNA }\end{array}$} & \multicolumn{3}{|c|}{$\begin{array}{l}\text { Number of probes within the imbalance detected } \\
\text { with amplified single cell DNA }\end{array}$} \\
\hline & & BAC clones & $\begin{array}{l}\text { SNP probes } \\
\text { (copy number) }\end{array}$ & $\begin{array}{l}\text { SNP probes } \\
\quad(\mathrm{LOH})\end{array}$ & BAC clones & $\begin{array}{l}\text { SNP probes } \\
\text { (copy number) }\end{array}$ & $\begin{array}{l}\text { SNP probes } \\
\quad(\mathrm{LOH})\end{array}$ \\
\hline \multirow[t]{2}{*}{$47, X Y,+21$} & Trisomy 21 & 35 & 3,935 & NR & 35 & 3,704 & NR \\
\hline & Monosomy X & 154 & 5,609 & 5,615 & 149 & 5,705 & 5,624 \\
\hline \multirow[t]{3}{*}{ 46,XY,der(20),t(18;20)(p11.21;p13) } & 9.3 Mb 18pter dup & 14 & 1,090 & NR & 20 & 1,217 & NR \\
\hline & 1.7 Mb 20pter del & 3 & 168 & 0 & 6 & 48 & 0 \\
\hline & Monosomy X & 154 & 5,610 & 5,593 & 132 & 5,705 & 5,647 \\
\hline \multirow[t]{2}{*}{$46, X, \operatorname{der}(X), t(X ; 14)(q 21.1 ; q 12.2)$} & 47.5 Mb 14qter dup & 63 & 4,162 & NR & 62 & 4,544 & NR \\
\hline & $58 \mathrm{Mb}$ Xqter del & 70 & 2,384 & 2,395 & 91 & 2,768 & 2,406 \\
\hline \multirow[t]{4}{*}{$46, \mathrm{XX}, \operatorname{del}(18)\left(\mathrm{p} 11.21 \rightarrow\right.$ pter $^{\mathrm{a}}$} & $14 \mathrm{Mb}$ 18pter del & 22 & 1,599 & 1,604 & $19^{a}$ & $1,599^{a}$ & $1,603^{a}$ \\
\hline & & & & & 12 & 1,599 & 1,575 \\
\hline & & & & & 14 & 1,611 & 1,594 \\
\hline & & & & & 9 & 1,599 & 1,565 \\
\hline
\end{tabular}

${ }^{a}$ Four single lymphoblastoid cells of the same EBV-transformed cell line were analyzed on BAC and SNP array.

$\mathrm{NR}$, not relevant; pter, the terminal part of the $\mathrm{p}$ arm of a chromosome.

To validate this method, we amplified single-cell DNA from Epstein-Barr virus (EBV)-transformed lymphoblastoid cells derived from individuals with chromosomal imbalances and hybridized it to the BAC and SNP arrays (Supplementary Methods online). As a control, we hybridized genomic DNA isolated from multiple cells of the corresponding EBV-transformed lines to both platforms (Tables 1 and 2). In all single-cell analyses, we detected all known monosomies and trisomies as well as the segmental deletions and duplications, but no false positives (Fig. 1a-c, Table 1 and Supplementary Fig. 1 online). The breakpoints of the segmental aberrations detected with the single-cell amplified DNA were located within a 0-400 SNP-probe interval surrounding the breakpoint delineated with the corresponding nonamplified genomic DNA (Table 1). Moreover, all known hemizygous regions were independently identified by $\mathrm{LOH}$ (Fig. 1c), except for the 1.7-megabase ( $\mathrm{Mb}$ ) deletion on chromosome 20 (Table 1). Alignment of the single-cell genotype with the genotype obtained from the corresponding nonamplified genomic DNA showed accurate SNP-typing from single cells: of the SNPs typed in the single cells ( $>67 \%$ of the 262,264 SNPs), more than $80 \%$ matched perfectly, 1-10\% did not match and the remaining SNPs could not be typed (Table 2 ).

In summary, the combination of BAC and SNP array CNV screening allowed reliable detection of genomic imbalances in single cells.

\section{Fertilized oocytes are chromosomally balanced}

We aspirated 18 human fertilized oocytes in the pronuclear stage and successfully amplified and analyzed 15 by BAC array CGH (15 of 18;
$83 \%$ ). Seven did not pass quality control testing, leaving eight fertilized oocytes that we further analyzed by SNP arrays to assess the frequency of chromosomal imbalances.

Five of these eight fertilized oocytes were male. Hence, a monosomy for the X chromosome was detected. One fertilized oocyte contained two terminal segmental duplications (Supplementary Fig. 1) that were probably derived from a chromosome breakage event during a preceding meiotic cell division. Thus, seven out of eight fertilized oocytes $(87.5 \%)$ were chromosomally balanced.

\section{Complex chromosomal imbalances in embryos}

We separately aspirated 165 blastomeres derived from 14 3-day-old embryos (mean 6.9 blastomeres per embryo) and nine 4-day-old embryos (mean 7.6 blastomeres per embryo) immediately after preimplantation genetic diagnosis (PGD) without cryopreservation. All 146 (146 of 165; 88\%) successfully amplified single-cell DNAs were analyzed by BAC array CGH; 50 of the $146(34 \%)$ were excluded on the basis of quality control of our algorithm and 86 (59\%) were further analyzed on SNP arrays. For the remaining ten blastomeres (7\%), the amount of amplified DNA was insufficient for SNP analysis (Supplementary Table 1).

Stringent interpretation criteria ensured that the number of chromosomal imbalances was a minimum estimate. For each embryo, we generated a karyogram that showed the copy number states of the different chromosomes per sister blastomere which we arbitrarily lettered (Fig. 2 and Supplementary Figs. 1-3 online). Of the 23 embryos, two were normal diploid (embryos 2 and 6), one embryo

Table 2 Accuracy of SNP typing on single lymphoblastoid cells

\begin{tabular}{|c|c|c|c|c|c|}
\hline $\begin{array}{l}\text { Karyotype of the } \\
\text { lymphoblastoid cells }\end{array}$ & $\begin{array}{l}\text { Number of SNPS } \\
\text { called in reference } \\
\text { genomic DNA (\%) }\end{array}$ & $\begin{array}{c}\text { Number of SNPs } \\
\text { called in single-cell } \\
\text { DNA (\%) }\end{array}$ & $\begin{array}{l}\text { Number of correct } \\
\text { called single cell } \\
\text { SNPs (\%) }\end{array}$ & $\begin{array}{c}\text { Number of incorrect } \\
\text { called single-cell } \\
\text { SNPs (\%) }\end{array}$ & $\begin{array}{l}\text { Number of } \\
\text { undetermined } \\
\text { SNPs (\%) }\end{array}$ \\
\hline $47, X Y,+21$ & $229,292(87.4)$ & $202,324(77.1)$ & $178,684(88.3)$ & $7,567(3.7)$ & $16,073(7.9)$ \\
\hline $46, X Y, \operatorname{der}(20), t(18 ; 20)(p 11.21 ; p 13)$ & $231,203(88.2)$ & $191,638(73.1)$ & $156,807(81.8)$ & $19,636(10.2)$ & $15,195(7.9)$ \\
\hline 46,X, $\operatorname{der}(X), t(X ; 14)(q 21.1 ; q 12.2)$ & $226,972(86.5)$ & $196,285(74.8)$ & $167,794(85.5)$ & $11,886(6.1)$ & $16,605(8.5)$ \\
\hline \multirow[t]{4}{*}{$46, \mathrm{XX}, \operatorname{del}(18)\left(\mathrm{p} 11.21 \rightarrow\right.$ pter $^{\mathrm{a}}$} & $206,605(78.8)$ & $189,921(72.4)$ & $155,213(81.7)$ & $7,841(4.1)$ & $26,867(14.1)$ \\
\hline & & $176,535(67.3)$ & $152,283(86.3)$ & $3,293(1.9 \%)$ & 20,959 (11.9) \\
\hline & & $180,777(68.9)$ & $150,073(83.0)$ & $7,101(3.9)$ & $23,603(13.1)$ \\
\hline & & $176,911(67.5)$ & $141,667(80.1)$ & $10,723(6.1)$ & $24,521(13.9)$ \\
\hline
\end{tabular}

${ }^{\mathrm{a}}$ Four single lymphoblastoid cells of the same cell line were analyzed on BAC and SNP array. 
a

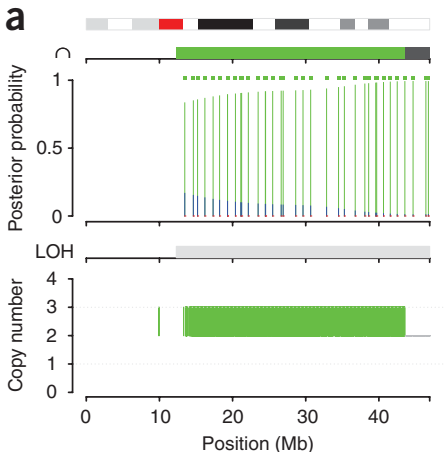

e
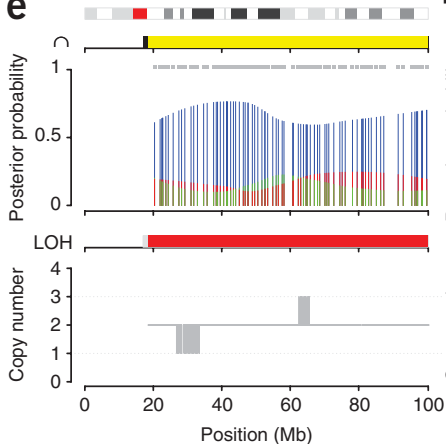

b
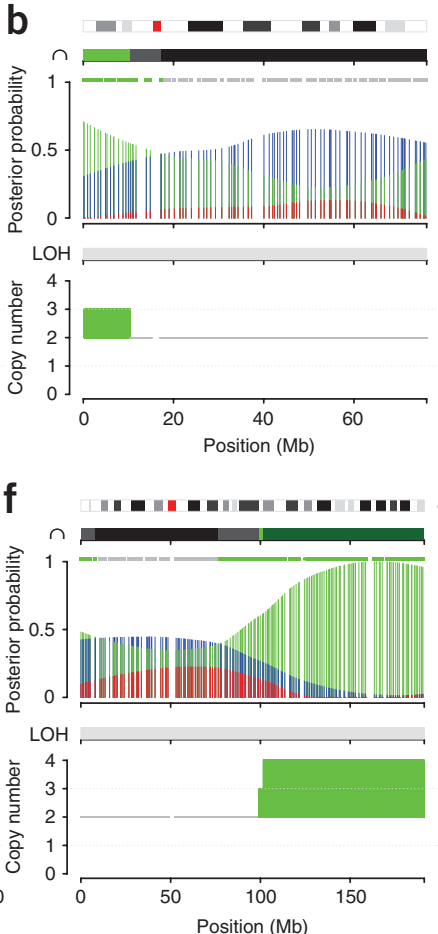
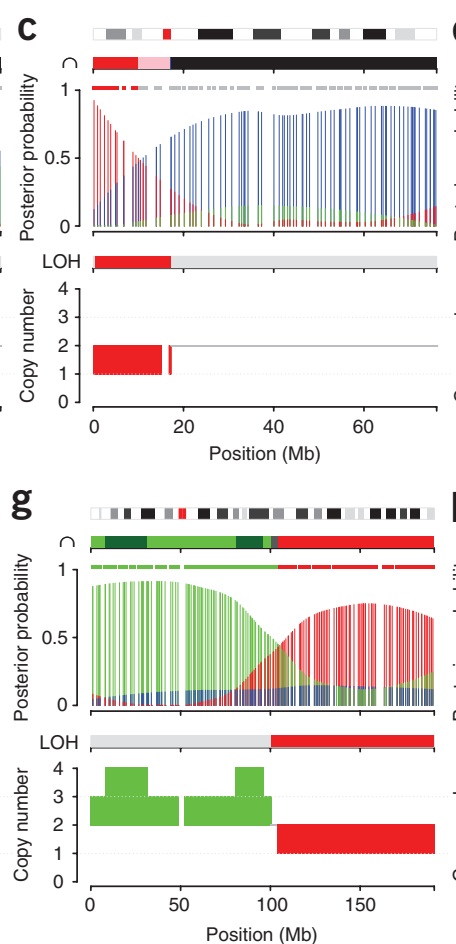
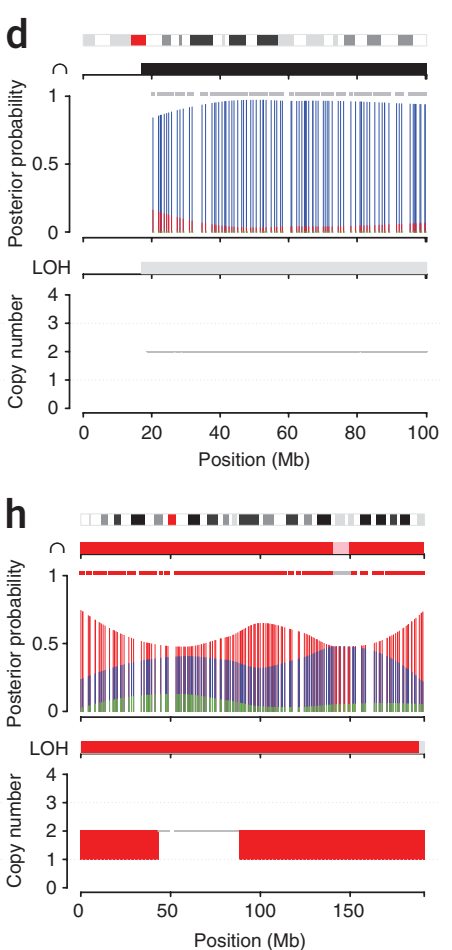

Figure 1 Genome-wide CNV and LOH detection in single cells. In each panel from top to bottom is shown a chromosome ideograph; a color consensus bar indicated with ' $\cap$ ' (black, green and red represent normal, duplicated and deleted regions, respectively; complete color legend in Supplementary Fig. 1); a bar indicating the highest smoothed posterior probability per BAC clone; the smoothed posterior probabilities per BAC clone for being normal (blue), deleted (red) and duplicated (green); the LOH segments indicated in red; and the SNP copy number status. (a-c) Aneuploidies in single lymphoblastoid cells: a trisomy 21 (a), a 9.3-Mb 18p duplication (b), a 14-Mb 18p deletion (c). (d-h) Status of specific chromosomes in single blastomeres of embryo 20.

(d) Normal chromosome 15 in blastomere d. (e) A UPID 15 in blastomere e. (f-h) Complex patterns of segmental imbalances. (f) A terminal 4q amplification in blastomere d. (g) A terminal $4 \mathrm{q}$ deletion and a duplication of the remaining chromosome 4 in blastomere $g$. The size of the deletion is identical to the size of the amplification in blastomere d. (h) A monosomy 4 in blastomere $\mathrm{c}$.

(embryo 8) was diploid but contained a de novo uniparental isodisomy (UPID), eight were mosaic, consisting of normal blastomeres as well as blastomeres with either whole-chromosome or segmental aneuploidies or both (embryos 3, 9, 11, 15-17, 21 and 23), in five embryos the normal diploid to aneuploid cell ratio was at least 1 (embryos 9, $11,15,21$ and 23), and twelve embryos were mosaic aneuploid, composed entirely of blastomeres with different whole-chromosome or segmental imbalances or both (embryos 1, 4, 5, 7, 10, 12-14, 18-20 and 22) (Fig. 2a).

Whole-chromosome imbalances were detected in 19 embryos (19 of 23; 83\%; embryos 1, 3-5, 7, 9-14 and 16-23). Within this group, only three embryos showed the same chromosome aneuploidy in all sister blastomeres (13\%), suggesting that they were caused by meiotic nondisjunction (trisomy 15 in embryo 18 (Fig. 2b), monosomy 20 in embryo 19 and monosomy 16 in embryo 14 (Supplementary Fig. 1)). All other imbalances resulted from mitotic segregation errors. Apart from whole-chromosome imbalances, mitotic malsegregations may also result in UPID. Chromosomes identified as diploid combined with chromosome-wide LOH are UPIDs. De novo UPIDs were detected in two embryos (9\%; embryos 8 and 20). Embryo 20, a male embryo, contained in two of its blastomeres UPIDs for chromosomes 2 and 15 as well as a UPID X that had a complementary nullisomy $\mathrm{X}$ (Figs. 1d-e and 2c). The remaining six blastomeres were both diploid and heterozygous for chromosomes 2 and 15 as well as monosomic for the X chromosome. Comparison of the SNPs across all blastomeres showed that UPIDs 2 and 15 were of different parental origin in the two blastomeres (Supplementary Table 2 online). In addition, the
UPID 2 in blastomere e (Fig. 2c and Supplementary Fig. 3) carried a terminal 2q duplication. Thus far, UPIDs have been believed to occur via two-step events, either through a monosomic conception rescued by mitotic nondisjunction, through mitotic rescue of a mitotic trisomy or monosomy or through more complex events involving either isochromosome formations, nonhomologous Robertsonian translocations or other translocations accompanied by the loss of the other allele ${ }^{17,18}$. The data presented here suggest that UPIDs can also occur by a single nondisjunction event of both replicated homologous chromosomes that are pulled towards opposite spindle poles during mitosis. This mitotic segregation pattern is reminiscent of the reductional cell division of meiosis I.

To obtain a view of the segmental imbalances across the blastomeres of a given embryo, we hereafter discuss only the 12 embryos for which two-thirds or more of the blastomeres were informative on both SNP and BAC arrays (embryos 1, 6, 8-10, 14, 15 and 17-21). We detected terminal segmental chromosomal imbalances in seven of the twelve embryos (58\%), and we classified them as simple or complex imbalances. Simple terminal imbalances represented terminal deletions, duplications or amplifications. In contrast, terminal imbalances were considered complex when accompanied by segmental aneuploidies for the remaining part of the chromosome. In some embryos, simple and complex terminal imbalances coexisted for different chromosomes.

We identified simple terminal imbalances for multiple chromosomes in six embryos (50\%; embryos 9, 10, 15 and 18-20). Embryo 18 contained a $1 \mathrm{p}$ terminal deletion in blastomere a (Fig. $\mathbf{2 b}$ and 


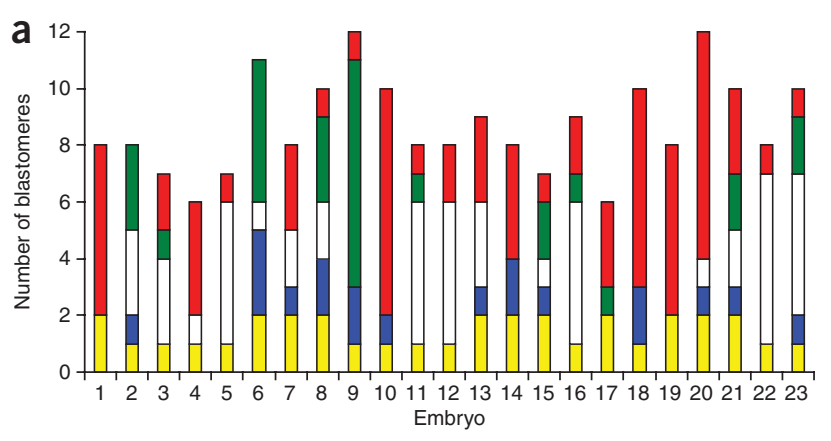

b
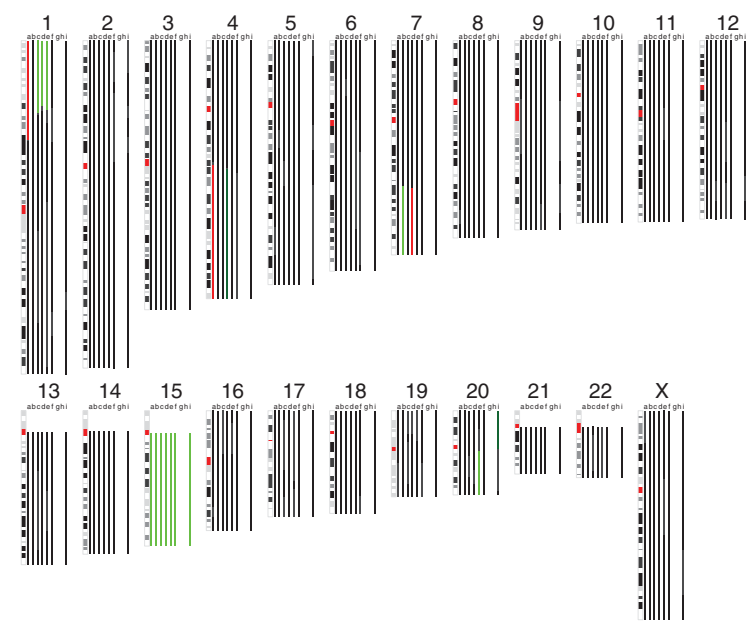

C
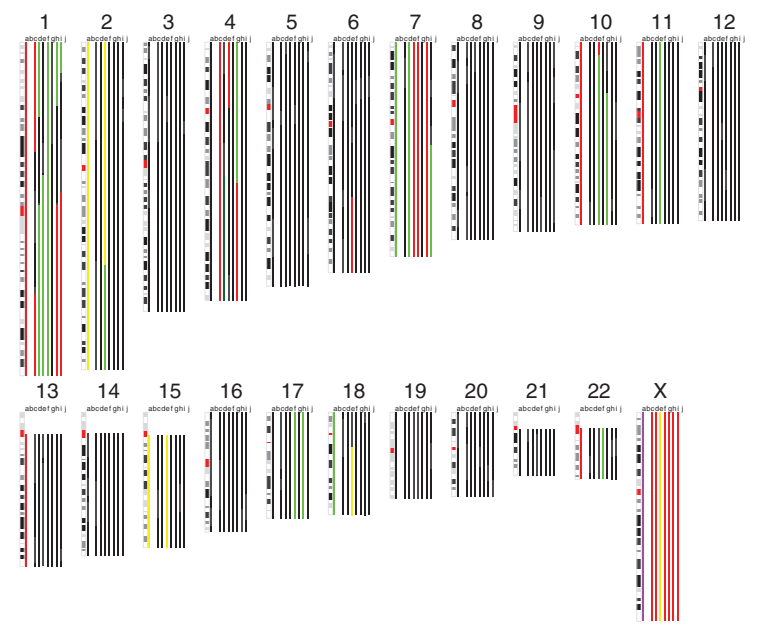

Supplementary Fig. 2) with a reciprocal duplication in three sister blastomeres (c, $\mathrm{d}$ and e), a 4q terminal deletion in blastomere a with a reciprocal amplification in blastomere $\mathrm{d}$ and a $7 \mathrm{q}$ terminal deletion in blastomere $\mathrm{d}$ with a reciprocal duplication in blastomere b (Fig. 2b and Supplementary Fig. 2). Similarly, in embryo 19, 4q and 10q terminal deletions with a reciprocal $4 \mathrm{q}$ duplication and $10 \mathrm{q}$ amplification were detected in a proportion of its sister blastomeres (Supplementary Fig. 1). For the other simple terminal imbalances, no reciprocal events were detected among the informative sister blastomeres.

We observed nine complex chromosomal imbalances in four of the twelve embryos (33\%; embryos 1, 10, 19 and 20). In embryo 20, we detected a $4 \mathrm{q}$ terminal deletion and a reciprocal $4 \mathrm{q}$ amplification in two blastomeres, whereas the remaining part of the chromosome proximal to the $4 \mathrm{q}$ deletion was trisomic (Figs. 1f,g and 2c). A sister
Figure 2 Compilation of CNV and LOH data of single blastomeres per embryo. (a) Per embryo, the number of blastomeres that were screened by PGD-FISH (yellow), insufficiently amplified for analysis (blue), did not pass the quality control (white), were chromosomally balanced (green), or were unbalanced or contained UPIDs (red). (b,c) Per embryo (embryo 18 (b), embryo 20 (c)) a karyogram composed of color bars that each represent the chromosome copy number state in a specific blastomere. Black represents a normal region, purple represents a homozygous deletion, red represents a hemizygous deletion, green represents a duplication, dark green represents an amplification, yellow represents a UPID and gray represents discordance between the analyses or unreliable aberrations.

blastomere containing a monosomy for chromosome 4 was identified, consistent with a mitotic nondisjunction event of the truncated chromosome 4 (Figs. 1h, 2c and 3). In addition, four remaining sister blastomeres contained two copies of chromosome 4 , whereas a fifth lacked a $4 \mathrm{p}$ arm (Fig. 2c). Comparable imbalances were observed for chromosome 10 in embryo 20 (Fig. 2c), chromosome 5 in embryo 19 , chromosome 4 in embryo 1 and chromosomes 4,15 and $\mathrm{X}$ in embryo 10 (Supplementary Fig. 1). Terminal imbalances associated with even more complex rearrangements were observed for chromosome 2 in embryo 10 and chromosome 1 in embryo 20 (Supplementary Fig. 1).

In addition to segmental terminal imbalances with random intrachromosomal breaks, a high number of imbalances showed breakpoints within the centromeric region. Whole-arm deletions or duplications were detected for multiple chromosomes in five embryos (42\%; embryos 10, 17, 18, 20 and 21).

\section{DISCUSSION}

Understanding the nature and incidence of chromosome instability during early embryogenesis provides insight into the causes of human infertility and into the mechanisms underlying constitutional chromosomal rearrangements. By developing a technology to assess genome-wide copy number variations and SNPs, we uncovered chromosomal rearrangements occurring at high frequency during early IVF embryogenesis but not in the preceding premeiotic or meiotic cell cycles. Segmental imbalances were observed in a staggering $70 \%$ of the embryos ( 16 of the 23 embryos analyzed and 9 of the 12 embryos with informative array results for at least two-thirds of the blastomeres) and could be classified in different patterns. Forty percent (9 of 23 and 5 of 12) carried entire chromosome-arm imbalances that might result from chromosome breakage or centric fission accompanied by malsegregation of the resulting telocentric or ring chromosomes or by the formation of isochromosomes $i(p)$ or $\mathrm{i}(\mathrm{q})^{19}$. Fifty-five percent of the embryos (12 of 23 and 7 of 12) carried terminal segmental imbalances that could be classified in simple and complex patterns and were probably the result of DNA doublestranded breaks followed by nondisjunction of the acentric fragment (Fig. 3). In seven of the nine complex terminal events, the terminal deletion was accompanied by a duplication of the remaining chromosomal segment. If such an imbalance was observed in one blastomere, an amplification of the corresponding deleted segment was often detected in a sister blastomere, whereas yet another sister blastomere was monosomic for the respective chromosome. This frequently observed complex pattern indicates that truncated chromosomes are often rescued by fusion of replicated sister chromatids resulting in dicentric isochromosomes. Monopolar segregation of the latter explains the complex patterns, whereas bipolar segregation yields apparently simple patterns characterized by breakage-fusion-bridge products with both pure terminal imbalances and terminal deletions accompanied by inverted duplications (Fig. 3). Exactly such 


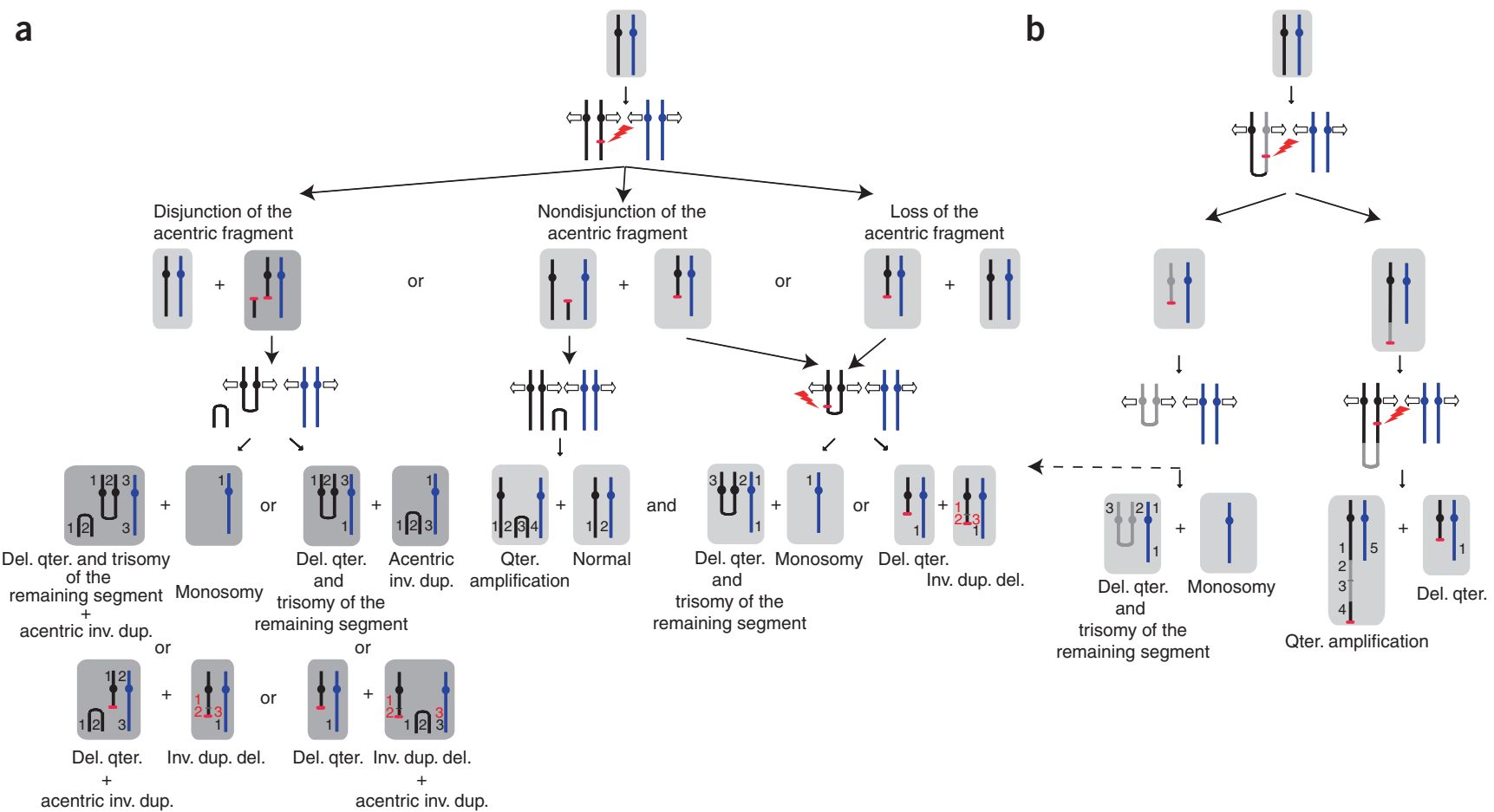

Figure 3 Genesis of simple and complex terminal imbalances. (a,b) A schematic showing the sequence of potential events downstream of a DNA doublestranded break (dashed arrow) (a) or telomere loss accompanied by breakage-fusion-bridge (BFB) cycles (b). Expected copy numbers are indicated. Both models predict the creation of dicentric inverted duplication (inv dup) isochromosomes by fusing replicated broken sister chromatids. According to our findings, such dicentric isochromosomes are frequently segregated to one spindle pole, resulting in a monosomy in the sister blastomere. However, if pulled toward opposite spindle poles, inverted duplication with terminal deletion (inv dup del) chromosomes as well as chromosomes with pure terminal deletions are generated. Amplified terminal segments are explained by a randomly segregating inv dup segment (a) or a second BFB cycle (b). Qter, the terminal part of the $\mathrm{q}$ arm of the chromosome.

chromosomal imbalances have recently been uncovered in individuals carrying chromosomal rearrangements; microscopic pure terminal deletions are often associated with submicroscopic inverted duplications $^{13}$ and ring chromosomes often contain inverted duplications flanking their fusion point ${ }^{14}$. The latter imbalances are thought to result from inverted duplication with terminal deletion (inv dup del) chromosomes that circularize for stabilization. Additionally, acentric marker chromosomes stabilized by neocentromere formation are found to be made up of inverted duplicated terminal chromosomal segments ${ }^{15}$. Notably, segmental aneuploidies resulting from breakage-fusion-bridge cycles are also observed in tumors ${ }^{20-22}$. Whether the molecular mechanisms underlying tumor and embryonic chromosome instability bear resemblance to each other remains to be established.

Although culture and stimulation conditions may influence the aneuploidy frequency in embryos ${ }^{23,24}$, there is no evidence for aberrant IVF culture conditions in this study. The baby-take-homerate for young couples (female age $<35$ years; mean $29.3 \pm 3.0$ years) with a PGD indication for sex selection or microdeletion screening is $23.3 \%$ at our fertility center (14 live births from 60 embryos transferred), which is in line with the most recent data of the European Society of Human Reproduction and Embryology PGD consortium ${ }^{25}$. Moreover, the aneuploidy rate observed here corresponded well with reports that use FISH aneuploidy screening on embryos from young women opting for $\mathrm{PGD}^{26-28}$ : by random selection of a single blastomere per embryo in our study and in silico determination of the copy number status of seven, nine or ten centromeric loci used in other studies, a mean aneuploidy rate of
$41 \%, 43 \%$ or $50 \%$, respectively, was observed, similar to published aneuploidy rates of $45 \%$ (ref. 26), $47.8 \%$ (ref. 27 ) and $64 \%$ (ref. 28).

Furthermore, the chromosome instability is unlikely to be caused by the genetic condition that is the reason for the PGD, as the 23 embryos were derived from nine couples each having a different genetic disorder (Supplementary Table 1), chromosome instability was observed in embryos from all nine couples, the mutations are inherited in mendelian frequencies if no PGD is applied, and, in four of the nine couples, the mutation was paternally inherited (Supplementary Table 1). As transcription is not activated until the 4- to 8-cell stage in human embryos ${ }^{29}$, only maternally inherited mutations would be expected to contribute to the chromosome instability phenotype in these cleavage-stage embryos.

As in vivo-conceived cleavage embryos are not available for research and embryos lost after the first week(s) of pregnancy are currently unattainable, the embryos used in this study are the best available representation of normal human embryogenesis. The chromosome instability observed in vitro most probably also occurs in vivo, as at most $30 \%$ of human conceptions result in a live birth ${ }^{30}$, more than $50 \%$ of spontaneous abortions have chromosomal imbalances ${ }^{31-33}$ and terminal deletions, duplications and isochromosomes, as well as mosaics, are frequently observed in live births ${ }^{34}$. These numbers corroborate our study, indicating that chromosome instability is prevalent in human embryogenesis. In addition to selection against chromosomally abnormal embryos, it seems probable that selection at the cellular level occurs against abnormal blastomeres. Only $9 \%$ of the embryos in this study were normal diploid in all blastomeres. However, IVF success rates after FISH-based PGD in our center and 
on average across IVF centers worldwide ${ }^{25}$ were higher than $20 \%$ per embryo transferred. Mosaic embryos containing normal blastomeres (39\% in this study) may thus result in chromosomally normal fetuses $^{35-37}$. Finally, the techniques developed in this study will allow for quantitative trait selection in IVF embryos and will prove invaluable for recording genomic changes in single cells during cell transformation, tumor progression and development of other human tissues ${ }^{1,38}$.

\section{METHODS}

Patient selection. We asked couples entering the IVF-PGD program for sex selection owing to $\mathrm{X}$-linked disorders or a $B R C A 2$ mutation or for familial microdeletion syndromes to participate (Supplementary Table 1). The inclusion criteria were written informed consent from both partners of each couple, a maternal age $\leq 35$ years (Supplementary Table 1), normal karyotype of both partners, a maternal body mass index of 18-30, initial normal semen parameters according to World Health Organization regulation, fresh (not cryopreserved) and good-quality embryos (that is, 2-5 blastomeres on day 2 after fertilization, $6-10$ blastomeres on day 3 after fertilization, $<20 \%$ fragmentation and equal-sized blastomeres) and no recurrent miscarriages. The study was approved by the Ethical Committee of the University Hospital Leuven.

Statistical analysis of bacterial artificial chromosome arrays. We analyzed individual array data by fitting a finite mixture of three normal distributions (N) per chromosome, with means of $0.5,0$ and -0.8 for the respective duplication, normal and deletion groups. We corrected the systematic biases by an estimated clone-specific mean and variance derived from a reference set of normal chromosomes constructed per cell type (that is, lymphoblastoids and blastomeres).

If $y_{\mathrm{jk}}$ is the mean $\log _{2}$ ratio value of the two spots available for clone $\mathrm{k}$ of chromosome $j$, then

$$
\begin{aligned}
y_{\mathrm{jk}} \approx & \pi_{\mathrm{dup}} N\left(0.5-\hat{\mu}_{\mathrm{jk}}, \hat{\sigma}_{\mathrm{jk}}^{2} / n_{\mathrm{jk}}\right)+\pi_{\mathrm{norm}} N\left(0-\hat{\mu}_{\mathrm{jk}}, \hat{\sigma}_{\mathrm{jk}}^{2} / n_{\mathrm{jk}}\right) \\
& +\pi_{\mathrm{del}} N\left(-0.8-\hat{\mu}_{\mathrm{jk}}, \hat{\sigma}_{\mathrm{jk}}^{2} / n_{\mathrm{jk}}\right),
\end{aligned}
$$

where $\mu_{\mathrm{jk}}$ is the estimated clone-specific mean, $\hat{\sigma}_{\mathrm{jk}}^{2}$ is the estimated clonespecific variance, $n_{\mathrm{jk}}$ is the number of observations (one or two observations) for clone $\mathrm{k}$ and $\pi_{\mathrm{dup}}, \pi_{\mathrm{norm}}$ and $\pi_{\mathrm{del}}$ are the a priori proportions for the respective groups.

We obtained the maximum likelihood estimates of the model parameters by the expectation-maximization algorithm. The a posteriori probabilities for each clone belonging to the duplication, normal and deletion group were calculated per chromosome and loess-smoothed with a span of $75 \%$ of the clones per chromosome to detect regions of successive clones belonging to a respective group. We performed data analysis by $\mathrm{R}$ (http://www.R-project.org/) and Matlab (MathWorks).

Optimization of the bacterial artificial chromosome algorithm. We constructed cell type-specific reference models for single-cell BAC array analyses, as multiple displacement amplification (MDA) is cell type dependent ${ }^{10}$. For the lymphoblastoids, we determined a reference set of normal chromosomes for mixture model building on the basis of karyotyping. Subsequently, we validated these reference chromosomes individually against the mixture model; only those that scored normal were retained as reference chromosomes. For the blastomere analysis, we selected for each chromosome at random 25 reference blastomeres on the basis of the SNP data. We used $\log _{2}$ ratios of the BAC array of these chromosomes to construct the mixture model. We validated these reference chromosomes individually against the model; only normals were retained as reference chromosomes.

Scoring criteria. We did not study single chromosomes if fewer than 18 BAC clones (that is, half the clones of the smallest chromosome) had signal intensities twofold above the autosomal median background intensity. Moreover, we considered single cells with genome-wide equal probabilities for the three copy number states per BAC clone as not informative, and we excluded them from further analyses on the basis of the quality control of our algorithm. These equal probabilities most likely originate from high standard deviations between intensity ratios detected on consecutive BAC clones. The reasons for equal probabilities are nontechnical, as deduced from the minor standard deviations between the duplicate spots and seem more likely to be biological. For instance, determination of copy numbers in cells during S-phase will inevitably lead to more scatter of intensity ratios detected on consecutive BAC clones. Alternatively, MDA efficiency might fluctuate more across the genome when chromosomes are highly condensed, as in the M-phase, with specific regions amplifying better than others. Furthermore, GC-rich regions (for example, chromosomes 17, 19 and 22) amplify less efficiently by MDA and are detected as potential deletions by SNP copy number analysis, most likely owing to referencing to nonamplified genomic DNA analyses. Nondeleted GCrich domains do not show $\mathrm{LOH}$ and are detected as diploid regions in the BAC analyses because of the correction for systematic MDA bias. Finally, in the blastomeres, we interpreted only imbalances comprising at least ten consecutive BAC probes after single-cell BAC array CGH analysis or at least 1,090 SNP probes after SNP copy number analysis. We based the assignment of a chromosome or chromosomal segment to be either duplicated or deleted on stringent interpretation criteria: for single events without reciprocal imbalances BAC array CGH, SNP-copy number and LOH needed to be concordant; for reciprocal copy number states in sister blastomeres, a false negative for either the BAC array CGH or SNP-copy number or LOH technique was tolerated; to score interstitial imbalances, BAC array CGH, SNP copy number and $\mathrm{LOH}$ needed to be concordant and a reciprocal segment had to be present in a sister blastomere.

Additional methodology. The collection of lymphoblastoids, fertilized oocytes and blastomeres, as well as the single cell array protocols, can be found in the Supplementary Methods.

Gene Expression Omnibus accession number. The data discussed in this publication have been deposited in the US National Center for Biotechnology Information Gene Expression Omnibus (GEO) and are accessible through GEO series accession number GSE11663. The GEO accession numbers of the platforms used are GPL6928, GPL6929 and GPL3718.

Note: Supplementary information is available on the Nature Medicine website.

\section{ACKNOWLEDGMENTS}

We thank all families who participated in the study, the Leuven University Fertility Center for technical assistance and S. Jackmaert for her help with the SNP arrays. We are also grateful to the Mapping Core and Map finishing groups of the Wellcome Trust Sanger Institute for initial BAC clone supply and verification and to the microarray facility of the Flanders Interuniversity Institute for Biotechnology for their help in spotting the arrays. We would like to thank C. Spiessens, E. Legius, T. de Ravel de l'Argentière, H. van Esch and K. Devriendt for the critical reading of the manuscript. This work was made possible by grants from the Institute for the Promotion of Innovation through Science and Technology (IWT-Flanders) (SBO-60848) and GOA/2006/12 and Center of Excellence SymBioSys (Research Council K.U.Leuven EF/05/007) to J.R.V. and Fonds de la Recherche Scientifique to M.V. E.V. was supported by the Institute for the Promotion of Innovation through Science and Technology in Flanders.

\section{AUTHOR CONTRIBUTIONS}

E.V., T.V. and J.R.V. designed the experiments and wrote the manuscript. E.V., T.V., C.L.C. and C.M. conducted the experiments. M. Ampe, G.V., P.K. and Y.M. developed the statistical algorithms and software for data analysis. M. Amyere, M.V. and F.S. provided expertise on SNP typing. J.-P.F. performed the genetic counseling of the couples opting for PGD. T.D. and S.D. performed IVF and provided the embryos. J.R.V. supervised.

Published online at http://www.nature.com/naturemedicine/

Reprints and permissions information is available online at http://npg.nature.com/ reprintsandpermissions/

1. Geigl, J.B., Obenauf, A.C., Schwarzbraun, T. \& Speicher, M.R. Defining 'chromosomal instability'. Trends Genet. 24, 64-69 (2008).

2. Lengauer, C., Kinzler, K.W. \& Vogelstein, B. Genetic instability in colorectal cancers. Nature 386, 623-627 (1997).

3. Gao, C. et al. Chromosome instability, chromosome transcriptome, and clonal evolution of tumor cell populations. Proc. Natl. Acad. Sci. USA 104, 8995-9000 (2007). 
4. Rajagopalan, H. \& Lengauer, C. Aneuploidy and cancer. Nature 432, 338-341 (2004).

5. Delhanty, J.D. Mechanisms of aneuploidy induction in human oogenesis and early embryogenesis. Cytogenet. Genome Res. 111, 237-244 (2005).

6. Voullaire, L., Slater, H., Williamson, R. \& Wilton, L. Chromosome analysis of blastomeres from human embryos by using comparative genomic hybridization. Hum. Genet. 106, 210-217 (2000).

7. Wells, D. \& Delhanty, J.D. Comprehensive chromosomal analysis of human preimplantation embryos using whole genome amplification and single cell comparative genomic hybridization. Mol. Hum. Reprod. 6, 1055-1062 (2000).

8. Daphnis, D.D. et al. Analysis of the evolution of chromosome abnormalities in human embryos from Day 3 to 5 using CGH and FISH. Mol. Hum. Reprod. 14, 117-125 (2008).

9. Harper, J. et al. What next for preimplantation genetic screening? Hum. Reprod. 23, 478-480 (2008).

10. Le Caignec, C. et al. Single-cell chromosomal imbalances detection by array $\mathrm{CGH}$. Nucleic Acids Res. 34, e68 (2006).

11. Fiegler, H. et al. High resolution array-CGH analysis of single cells. Nucleic Acids Res. 35, e15 (2007).

12. Iwamoto, K. et al. Detection of chromosomal structural alterations in single cells by SNP arrays: a systematic survey of amplification bias and optimized workflow. PLoS One 2, e1306 (2007).

13. Ballif, B.C., Yu, W., Shaw, C.A., Kashork, C.D. \& Shaffer, L.G. Monosomy 1p36 breakpoint junctions suggest pre-meiotic breakage-fusion-bridge cycles are involved in generating terminal deletions. Hum. Mol. Genet. 12, 2153-2165 (2003).

14. Rossi, E. et al. Duplications in addition to terminal deletions are present in a proportion of ring chromosomes. Clues to the mechanisms of formation. J. Med. Genet. 45, 147-154 (2008)

15. Marshall, O.J., Chueh, A.C., Wong, L.H. \& Choo, K.H. Neocentromeres: new insights into centromere structure, disease development, and karyotype evolution. Am. J. Hum. Genet. 82, 261-282 (2008).

16. Ballif, B.C. et al. Detecting sex chromosome anomalies and common triploidies in products of conception by array-based comparative genomic hybridization. Prenat. Diagn. 26, 333-339 (2006).

17. Kotzot, D. Abnormal phenotypes in uniparental disomy (UPD): fundamental aspects and a critical review with bibliography of UPD other than 15. Am. J. Med. Genet. 82, 265-274 (1999).

18. Kotzot, D. Complex and segmental uniparental disomy (UPD): review and lessons from rare chromosomal complements. J. Med. Genet. 38, 497-507 (2001).

19. Perry, J., Slater, H.R. \& Choo, K.H. Centric fission—simple and complex mechanisms. Chromosome Res. 12, 627-640 (2004).

20. Pipiras, E., Coquelle, A., Bieth, A. \& Debatisse, M. Interstitial deletions and intrachromosomal amplification initiated from a double-strand break targeted to a mammalian chromosome. EMBO J. 17, 325-333 (1998).
21. Artandi, S.E. et al. Telomere dysfunction promotes non-reciprocal translocations and epithelial cancers in mice. Nature 406, 641-645 (2000).

22. Bignell, G.R. et al. Architectures of somatic genomic rearrangement in human cancer amplicons at sequence-level resolution. Genome Res. 17, 1296-1303 (2007).

23. Munné, S. et al. Treatment-related chromosome abnormalities in human embryos. Hum. Reprod. 12, 780-784 (1997).

24. Weghofer, A. et al. The impact of LH-containing gonadotropins on diploidy rates in preimplantation embryos: long protocol stimulation. Hum. Reprod. 23, 499-503 (2008).

25. Harper, J.C. et al. ESHRE PGD consortium data collection VII: cycles from January to December 2004 with pregnancy follow-up to October 2005. Hum. Reprod. 23, 741-755 (2008).

26. Rubio, C. et al. Chromosomal abnormalities and embryo development in recurrent miscarriage couples. Hum. Reprod. 18, 182-188 (2003).

27. Munné, S. et al. Increased rate of aneuploid embryos in young women with previous aneuploid conceptions. Prenat. Diagn. 24, 638-643 (2004).

28. Baart, E.B. et al. Preimplantation genetic screening reveals a high incidence of aneuploidy and mosaicism in embryos from young women undergoing IVF. Hum. Reprod. 21, 223-233 (2006).

29. Braude, P., Bolton, V. \& Moore, S. Human gene expression first occurs between the four- and eight-cell stages of preimplantation development. Nature 332, 459-461 (1988).

30. Macklon, N.S., Geraedts, J.P. \& Fauser, B.C. Conception to ongoing pregnancy: the 'black box' of early pregnancy loss. Hum. Reprod. Update 8, 333-343 (2002).

31. Pflueger, S. Cytogenetics of spontaneous abortion. in The Principles of Clinical Cytogenetics (eds. Gersen, S. \& Keagle, M.) 317-343 (Humana Press, New Jersey, 1999).

32. Fritz, B. et al. Cytogenetic analyses of culture failures by comparative genomic hybridisation (CGH) - re-evaluation of chromosome aberration rates in early spontaneous abortions. Eur. J. Hum. Genet. 9, 539-547 (2001).

33. Benkhalifa, M. et al. Array comparative genomic hybridization profiling of firsttrimester spontaneous abortions that fail to grow in vitro. Prenat. Diagn. 25, 894-900 (2005).

34. Schinzel, A. Catalogue of Unbalanced Chromosome Aberrations in Man (Walter de Gruyter, New York, 2001).

35. Staessen, C. et al. Comparison of blastocyst transfer with or without preimplantation genetic diagnosis for aneuploidy screening in couples with advanced maternal age: a prospective randomized controlled trial. Hum. Reprod. 19, 2849-2858 (2004).

36. Li, M. et al. Fluorescence in situ hybridization reanalysis of day- 6 human blastocysts diagnosed with aneuploidy on day 3. Fertil. Steril. 84, 1395-1400 (2005).

37. Munné, S. et al. Self-correction of chromosomally abnormal embryos in culture and implications for stem cell production. Fertil. Steril. 84, 1328-1334 (2005).

38. Yurov, Y.B. et al. Aneuploidy and confined chromosomal mosaicism in the developing human brain. PLoS One 2, e558 (2007) 